\title{
Análise de redes sociais aplicadas ao transporte aeroviário de cargas
}

\author{
João Pedro Pinheiro Malere', Vladimir Minas² e Giovanna Miceli Ronzani Borille ${ }^{3}$
}

\begin{abstract}
Resumo: O presente artigo possui como objetivo analisar redes de transporte aéreo exclusivamente de carga nos Estados Unidos durante uma década, entre os anos de 2004 e 2014. Para isso é utilizada a Análise de Redes Sociais onde indicadores como closeness (centralidade de proximidade) e betweenness (centralidade de intermediação) são calculados. O artigo esboça comparações entre as redes e respectivas métricas das duas maiores empresas aéreas do setor e a das 18 maiores aéreas seguintes, o que permite avaliar o impacto de retrações econômicas, como a ocorrida em 2008, nessas redes assim como revelar assimetrias entre empresas de diferentes portes. Pode-se notar que, dentre outros aspectos, o grafo do transporte aéreo de carga gerado apresenta grande influência das duas maiores empresas privadas do setor, a FedEx e a UPS o que pode ser evidenciado, por exemplo, pelo número de nós que, em 2014, apresentou os valores de 178 e 106 respectivamente em comparação com 81 das demais 18 maiores aéreas.

Palavras-chave: redes de transporte aéreo de carga; análise de redes sociais; redes complexas.
\end{abstract}

Abstract: The present work objective is the analysis of the United States air cargo transport during a decade, from the year 2004 to 2014. The network theory is used and indicators such as closeness and betweenness are calculated. The present work compares the networks and the respective metrics of the two main airlines of the industry and the other 18 biggest companies what enables the evaluation of the impact of economic recessions, such as the one from 2008, on these networks and the detection of assymetries between companies of different sizes. It is possible to note that, among other aspects, the air cargo transport graph is heavily influenced by the two main private companies of the sector, FedEx and UPS, what can be pointed out by, e.g., the number of nodes of 178 and 106 in 2014 respectively for these networks compared to 81 from the other 18 biggest companies.

Keywords: cargo air transportation networks; social network analysis; complex networks.

\section{INTRODUÇÃO}

O transporte aeroviário de cargas tem sido o principal modo de transporte escolhido por agentes econômicos que buscam soluções logísticas focadas na rapidez e na confiabilidade da movimentação de produtos dentro do mercado doméstico e internacional (Grandjot et al., 2007), ampliando sua importância em um contexto de crescente globalização, especialização da economia produtiva e de adoção constante de estratégias empresariais com ênfase na redução dos níveis de estoques e prazos de entrega (Scholz, 2012).

Assim como qualquer outro modo de transporte, como o rodoviário, o aquaviário e o ferroviário, o meio aéreo é influenciado pelo desenvolvimento da economia mundial e possui correlação com o Produto Interno Bruto - PIB. O setor de transporte de cargas aéreas no mundo, que vinha crescendo desde de 1983 a uma taxa anual média de 5,2\%, com base na receita tonelada-quilômetros (RTK - Revenue Tonne-Kilometers), foi afetado pela crise econômica global deflagrada em 2008, passando por um longo período de recessão e estagnação. $\mathrm{O}$ volume de receita no modo aéreo em 2008 e 2009 foi reprimido em 13\% e após a recuperação em 2010 empresas e entidades especializadas no transporte aeroviário projetaram para o setor um crescimento médio

\footnotetext{
1 João Pedro Pinheiro Malere, ITA. (jpmalere@gmail.com)

2 Vladimir Minas, Departamento de Engenharia, ITA. (mvladi@ig.com.br)

${ }^{3}$ Giovanna Miceli Ronzani Borille, Divisão de Engenharia Civil, ITA.

(ronzani@ita.br)
}

Manuscrito recebido em 30/03/2016 e aprovado para publicação em 07/09/2016

Este artigo é parte de TRANSPORTES v. 24, n. 4, 2016. ISSN: 2237-1346 (online). DOI:10.14295/transportes.v24i4.1096 anual de 4,7\% nos próximos 20 anos, a partir de 2014 (BOEING, 2014).

Dado o supracitado, o presente trabalho concentra-se na exploração de ferramentas e métodos para identificar e avaliar características específicas do setor de transporte aéreo de cargas, tendo como objetivo analisar a influência das maiores corporações do setor a fim de, por exemplo, subsidiar ações de planejamento para essa opção logística de progressiva importância. É utilizado o processo de Análise de Redes Sociais, conceitualmente desenvolvido no campo da sociologia, psicologia social e antropologia e que compreende o estudo de uma rede e a respectiva relação entre os conjuntos de atores, os tipos de conexões e estruturas existentes e a compreensão de fatores relevantes para um sistema específico de transporte aéreo de cargas no contexto econômico de um país.

A partir dos métodos de Análise de Redes Sociais, o setor de transporte aeroviário de cargas do mercado doméstico dos Estados Unidos é analisado empregando-se um conjunto de métricas. Utilizaram-se datasets disponibilizados pelo United States Department of Transportation relativos à janela de tempo entre os anos de 2004 e 2014 contendo dados sobre o tráfego aéreo de passageiros e cargas no mercado doméstico do país de análise.

Quando se avalia o sistema de transporte aéreo nos Estados Unidos de forma integral, passageiros e carga, verifica-se que é formado por uma rede ampla, constituída de cerca de 850 aeroportos, dezenas de companhias aéreas e milhares de voos (Couto et al., 2015), tendo essa rede evoluído desde a desregulamentação do setor após 1978 de um sistema predominante de rotas lineares "ponto a ponto" para estruturas do tipo hub and spoke, com diversas conexões a partir da introdução de grandes aeronaves (wide body air- 
craft), além da introdução de companhias aéreas que operam com baixo custo e tarifas (low-cost airlines - LCCs) (Cook e Goodwin, 2008).

Para o presente estudo de fatores determinantes que moldam de maneira mais restritiva as características do sistema destinado ao transporte de cargas, exigiu-se a estratificação e organização dos dados em dois conjuntos distintos, sendo: (i) estrutura especificamente relacionada ao transporte de cargas, operada por aeronaves configuradas para o transporte exclusivo de bens e mercadorias (Cargo Aircraft); e (ii) Estrutura relacionada ao transporte misto de passageiros e cargas, operada por aeronaves combinadas (Combi Aircraft- Combined Passenger and Cargo Aircraft) que podem utilizar o deck principal atrás dos passageiros e o porão para transporte de carga paga ou por aeronaves que transportam exclusivamente passageiros (Passenger Aircraft) e que utilizam o espaço não ocupado por bagagens no porão para transporte de carga paga (belly cargo). Com base nessa distinção e com o propósito de avaliar a evolução das métricas ao longo de mais de uma década, utilizaram-se $d a$ tasets entre os anos de 2004 a 2014 referentes à estrutura que abrange a operação de Cargo Aircraft. Apesar do foco estar no transporte exclusivo de carga, comparações com o transporte aéreo como um todo, assim como outras modalidades, são realizadas em algumas situações onde são consideradas pertinentes, como na evolução do número de aeroportos em geral e aqueles específicos para carga, assim como na comparação do volume transportado por aeronaves e por outras modalidades de transporte.

\section{DESENVOLVIMENTO}

\subsection{Características do setor de transporte aeroviário de cargas}

Embora a participação do transporte aeroviário seja relativamente pequena dentro da matriz de transportes de cargas, quando medida em tonelada-quilômetros transportada, sua importância para a cadeia de suprimentos é elevada em razão do alto valor dos bens e mercadorias movimentados. Estimativas apontam que o transporte aéreo de cargas corresponda a $1 \%$ do mercado mundial em termos de toneladas transportadas e a $35 \%$ quando se estabelece como parâmetro o valor dos produtos conduzidos (BOEING, 2014).

Nesse modo de transporte sobressaem os aspectos relacionados à velocidade, segurança e comodidade, sendo vantajoso para distâncias médias e longas (Batti e Faria, 2011). Devido ao maior custo é mais adequado para o transporte de mercadorias de alto valor agregado e produtos perecíveis. Outras vantagens podem ser apontadas como a flexibilidade, pontualidade, tempos reduzidos de frete, possibilitando redução de níveis de estoques e, consequentemente, menores custos, e ampla cobertura geográfica. Desenvolveu-se originalmente como um subproduto do transporte de passageiros desempenhado pelas companhias áreas, tornando-se um negócio robusto e até independente com a utilização de aeronaves exclusivas para o transporte de cargas e oferta de serviços especializados (Scholz, 2012).

Basicamente subdivide-se em duas modalidades predominantes onde em uma categoria as cargas são movimentadas como um subproduto do transporte de passageiros, com a utilização pelas companhias áreas de aeronaves configuradas tanto para o transporte de passageiros, quanto para o transporte de cargas (Combi aircraft). A outra modalidade diz respeito à utilização de aeronaves exclusivamente configuradas, produzidas ou convertidas, para o transporte de bens e mercadorias (Cargo aircraft), compondo a outra categoria. Estimativas apontam que a subdivisão do mercado mundial do transporte aeroviário de cargas seja igualmente distribuída entre essas duas categorias (Bowen, 2004). Para o mercado dos EUA, a tendência é de concentração do volume transportado em voos dedicados à carga, o que corresponde a cerca de $80 \%$ do volume transportado em 2012, de acordo com Donatelli (2012).

O modelo de negócios que envolve o sistema aeroviário exclusivo de transporte de passageiros, incluindo as questões relacionadas ao tipo de aeronaves e infraestrutura aeroportuária, é centrado nas necessidades dos passageiros e venda de passagens. Quanto ao sistema exclusivo de transporte de cargas, fatores como a dimensão, volume, peso da carga e necessidades de apoio à operação nos aeroportos tornam-se relevantes. Ou seja, os dois modelos, apesar de semelhantes, envolvem variáveis bastante distintas no planejamento dos negócios.

Scholz (2012) aponta que o sistema de transporte de cargas realizado em aeronaves compartilhadas (Combi aircraft) é formatado essencialmente para atender os aspectos relativos ao transporte de passageiros, desde rotas, aeroportos e aeronaves. Por outro lado, o sistema operado por aeronaves de carga possui maior independência, podendo a companhia oferecer serviços mais especializados e estabelecer voos regulares com rotas, aeronaves e horários préprogramados dentro de um período específico, como por semana, ou voos charter onde o cliente escolhe, dentre outras variáveis, a origem, destinação, aeronaves e prazos específicos.

O transporte de cargas aéreo exige a integração com outras modalidades de transporte para que as mercadorias possam ser levadas da origem ao destino final. Por conseguinte, está organizado por diferentes etapas que abrangem primordialmente a coleta no cliente, a consolidação e preparação da carga, o transporte para o aeroporto de origem e abastecimento da carga na aeronave, o voo, a retirada da carga da aeronave no aeroporto de destino, a desconsolidação da carga e finalmente o transporte e entrega para o destinatário final (Scholz, 2012). No que diz respeito ao transporte aéreo de carga nos Estados Unidos, destacam-se as duas empresas que possuem operações integradas, Fedex e UPS (Donatelli, 2012). Ainda de acordo com Donatelli (2012), nas empresas integradas as aeronaves coletam a carga durante a noite nos hubs principais e se dirigem para as localidades mais próximas aos destinos finais, onde caminhões e outros veículos finalizam o processo de entrega. A aeronave aguarda para a coleta de novas cargas até o fim do dia, onde retorna novamente para o $h u b$, repetindo o ciclo.

De acordo com Helmig (2005), a etapa relacionada ao voo, ou em outras palavras o frete aéreo propriamente dito, corresponde usualmente em média a apenas $17 \%$ do tempo total do transporte.

No que se refere especificamente ao país levado em consideração para esse estudo (EUA), nota-se a presença de duas grandes empresas no setor de cargas que, de acordo 
com os dados analisados na seção 2.4 , correspondem a aproximadamente $80 \%$ do volume transportado, além de outras empresas com menor porte que possuem maior foco em regiões geográficas e tipo de carga transportada, dentre as quais se destacam as companhias Cathay Pacific Airways, Korean Air Lines e Atlas Air Inc., as maiores em 2014 em termos de carga transportadas após a Fedex e a UPS. Apesar das empresas Cathay e Korean serem respectivamente de Hong Kong e da Coreia as mesmas realizam voos domésticos exclusivos de carga dentro dos Estados Unidos.

Ao se analisar a rede de transporte aéreo de cargas sob a ótica da dinâmica econômica desse setor, alguns aspectos podem ser investigados, como: se há concentração de mercado devido à grande pressão por preços e aos ganhos de escala; e se a evolução da demanda, frente ao crescente comércio eletrônico ou flutuações do produto interno bruto, afeta de alguma maneira a configuração das redes das empresas. No presente trabalho somente um subconjunto desses aspectos serão investigados, como o impacto de retrações econômicas, como a de 2008, na rede de transporte de carga.

\subsection{A análise de redes sociais}

Scott e Carrington (2011) definem a Análise de Redes Sociais como a análise de sistemas de relações sociais representados por redes.

A Análise de Redes Sociais foi desenvolvida originalmente nos campos da sociologia, antropologia e psicologia, ganhando crescente interesse dos pesquisadores entre 1930 e 1970, quando foram concebidos os conceitos iniciais sobre "estrutura social", o formato e características das redes (networks), como a densidade e demais padrões de relações entre grupos e indivíduos (Scott, 1991). Dentro desse contexto também surgiu o sociograma, tido como a representação gráfica das interações sociais constituídas por linhas e pontos (Scott e Carrington, 2011). De 1970 em diante houve um grande incremento nas contribuições técnicas para o desenvolvimento do método de análise de redes sociais e suas aplicações, sendo constatada sua utilização em diversos campos como na Física e Economia (Scott e Carrington, 2011).

De acordo com o método, uma rede social refere-se a uma estrutura formada por pontos, chamados de vértices ou atores, que estão ligados por linhas chamadas de arestas e que representam alguma relação existente entre esses vértices. Esses atores podem representar pessoas, instituições, empresas, países; e as arestas representam o tipo de ligação existente entre eles, que pode ser de afinidade, troca de informação, materiais, documentos, entre outras (Wasserman e Faust, 1994).

Como um dos elementos centrais do processo de Análise de Redes Sociais está a Teoria de Grafos, originária da área de matemática e empregada no estudo das relações entre os objetos de um determinado conjunto, representado por pontos (vértices) e linhas (arestas). A Teoria de Grafos proporcionou as bases para a análise formal das propriedades dos sociogramas, auxiliando na avaliação dos resultados através da quantificação de medidas verificadas na rede, como o grau dos vértices, densidade da rede, medidas de centralidade, entre outras.
O estudo de redes, contudo, tem evoluído nos últimos anos mudando o foco da análise de simples grafos e respectivos vértices e arestas individualmente, para a avaliação em larga escala de propriedades estatísticas desses grafos, possibilitada nos dias atuais pela ampla disponibilidade de sistemas informatizados e redes de comunicação. Comumente são realizados estudos de redes complexas constituídas por milhões ou mesmo bilhões de vértices, o que claramente demanda uma mudança de postura analítica (Newman, 2003).

Um grafo (ou rede) consiste em um par ordenado $G$ $=(V(G), E(G))$, em que $V(G)$ é o conjunto de vértices e $E(G)$ é o conjunto de arestas que os conectam. Vértices conectados por uma aresta são chamados adjacentes. Denotase o número de vértices do grafo $G$ por $n$ e o número de arestas por $m$. Um grafo em que $m=0$ é denominado totalmente desconexo ou vazio (Cerqueira et al., 2014). As redes ainda podem ser ponderadas de forma a representar o grau de interação entre os vértices, representando ligações sociais fortes ou fracas (Newman, 2004).

A aplicação da Análise de Redes Sociais permite identificar e avaliar em uma rede a performance dos atores componentes com base na forma como interagem e, consequentemente, definem-se o grau de importância desses atores e a influência que exercem na rede. Aspectos estruturais como a existência ou não de grupos, a intensidade dos laços, a coesão e a vulnerabilidade da rede com base na atuação dos seus atores podem ser ainda observados e quantificados. Medidas estruturais e parâmetros topológicos de diferentes redes podem ser comparados entre si.

No presente artigo os aeroportos que formam o sistema aeroviário de transporte de cargas constituem os vértices da rede, as arestas representam os voos operados pelas linhas aéreas, definidos pelas rotas estabelecidas entre aeroportos. A utilização do método para avaliação do transporte aéreo de cargas possibilita identificar os aeroportos centrais e de maior importância para o sistema, bem como a maneira que as linhas aéreas operadoras de transporte de carga priorizam e organizam a infraestrutura existente para melhor condução de seus negócios.

\subsection{Métricas de rede}

A seguir são apresentados os conceitos relacionados ao conjunto de métricas de rede utilizadas no presente estudo, com base em Newman (2003), Reka e Barabási (2002) e Couto et al. (2015).

\subsubsection{Grau de entrada (in-degree) e grau de saída (out- degree)}

O Grau de Entrada de um nó (ou vértice) $v, k_{i n}(v)$, é dado pelo número total de ligações (arestas) que entram nesse nó. Da mesma forma, o Grau de Saída de um nó v, $k_{\text {out }}(v)$ é dado pelo número total de ligações que saem do nó. Assim, o grau de um nó $v, k(v)$, é obtido pelo somatório de $k_{\text {in }}(v)$ e $k_{\text {out }}(v)$. O Grau Médio, $\langle k\rangle$ de uma rede $G$ como mostra a Equação (1), é representado pela divisão entre a soma dos graus de todos os nós pelo número de vértices do grafo $(|\mathrm{V}|)$.

$$
<k>(G)=\sum_{\forall v \in V} \frac{k(v)}{|V|}
$$


Por intermédio de tais medidas, é possível obter uma indicação sobre os atores centrais em uma rede, considerando que atores centrais mostram-se mais ativos, apresentando maior número de ligações e grau mais elevado que os demais.

\subsubsection{Caminho mais curto (shortest path)}

Assumindo $\mathrm{P}_{\mathrm{u}, \mathrm{v}}$ como um conjunto de caminhos entre um dado par de nós $u$ e $v$, definimos o caminho mais curto $l(u, v)$ como sendo aquele que possui o menor número de "saltos" entre a origem e o destino. Temos igualmente $L$ como o conjunto de todos os caminhos mais curtos $l(u, v)$, $\forall(u, v)$ e o módulo de $L(|L|)$ o número de caminhos mais curtos do grafo. O caminho mais curto médio $\langle l\rangle$, de uma rede $G$ é obtido por:

$$
<l>(G)=\frac{1}{|L|} \sum_{\forall l(u, v) \in L} l(u, v)
$$

\subsubsection{Diâmetro}

Assumindo $l(u, v)$ como o caminho mais curto entre os nós $u$ e $v$, o diâmetro, $d$, é definido como o maior caminho mais curto entre qualquer par de nós da rede, sendo obtido por:

$$
d(G)=\max _{\forall(u, v) \in V} l(u, v)
$$

A propriedade do diâmetro proporciona uma noção sobre a dispersão na rede $G$.

\subsubsection{Coeficiente de agrupamento médio (clustering coefficient)}

O coeficiente de agrupamento é também conhecido como medida de transitividade da rede e representa a medida do grau com que os nós de um grafo tendem a agruparse. Em termos de topologia de uma rede, a transitividade representa que há a presença de um elevado número de triângulos nessa rede, ou seja, de três vértices conectados entre si. O coeficiente de agrupamento $C$ de uma rede $G$ com $n$ vértices é apresentado na Equação (4).

$$
\begin{gathered}
C(G)=\frac{1}{n} \sum_{i=1}^{n} C_{i} \\
C_{i}=\frac{\left|e_{j k}: v_{j}, v_{k} \in N_{i}, e_{j k} \in E\right|}{k_{i}\left(k_{i}-1\right)}
\end{gathered}
$$

Onde $e_{j k}$ representa as arestas que conectam os nós vizinhos ao nó $i, v_{j}$ e $v_{k}$ reprentam os vértices vizinhos ao nó $i, N_{i}$ define o grafo vizinho ao vértice $i$ e $k_{i}$ define o número de vizinhos do vértice $i$. Desse modo, podemos notar que o coeficiente de agrupamento funciona como uma medida da densidade de arestas estabelecidas entre os vizinhos de um nó.

\subsubsection{Densidade da rede (graph density)}

A densidade $D$ de uma rede é definida como a proporção entre o número de arestas $|E|$ e o número total de possíveis arestas, considerando a rede como um todo. A densidade da rede é dada por:

$$
D=\frac{2|E|}{|V|(|V|-1)}
$$

Conforme De Nooy et al. (2005), a densidade demonstra a coesão de uma rede, significando que quanto mais densa for uma rede, mais coesa ela será.

\subsubsection{Centralidade de intermediação (betweenness)}

A centralidade de intermediação ou betweenness $\beta$ de um nó $v$ é a fração dos caminhos mais curtos que conectam todos os pares de nós que passam por $v$. Ou seja, assumimos que $\sigma(j, k)$ representa o número de caminhos mais curtos entre os nós $j$ e $k$, e que $\sigma_{(j, k)}(v)$ representa o número daqueles caminhos que cruzam o nó $v$. O betweenness de um nó $v$ é dado por:

$$
\beta(v)=\sum_{j \neq v \neq k \forall V} \frac{\sigma_{(j, k)}(V)}{\sigma_{(j, k)}}
$$

O betweenness avalia em que medida um determinado ator em uma rede constitui-se como elo necessário entre diferentes grupos pertencentes à rede, levando em conta o menor caminho possível. De acordo com Freeman (1979), a centralidade de intermediação aborda a questão do controle que esses atores intermediários possuem sobre aqueles atores que dependem localmente desse intermediário.

\subsubsection{Centralidade de proximidade (closeness)}

A centralidade de proximidade ou closeness $\gamma$ de um nó $v$ identifica o quão próximo é esse nó dos demais nós possíveis de serem atingidos em uma rede. Tendo $\pi(v, k)$ como o comprimento do caminho mais curto entre um nó $v$ e qualquer outro nó $k$ possível de ser atingido, a centralidade de proximidade é definida como:

$$
\gamma(v)=\left[\sum_{k \neq v, k \in V} \pi(v, k)\right]^{-1}
$$

A centralidade de proximidade busca identificar a extensão em que um ator é observado como o centro da rede, se requisita poucos intermediários para contatar outros, posicionando-se estruturalmente de maneira independente

\subsubsection{PageRank}

Consiste em algoritmo utilizado pela ferramenta de busca Google para posicionar de forma hierárquica páginas de internet entre os resultados de sua busca. A base fundamental do método é constituída pela premissa de que nós mais importantes em uma rede receberão um número maior de ligações de outros nós (Page et al., 1998).

O Rank (hierarquia) de um nó $P i$ é dado pela soma do $R a n k$ de todos os nós que apontam para o nó $P i$, dividido pelo número de nós que o nó $P i$ aponta para, conforme: 


$$
r\left(P_{i}\right)=\sum_{P_{j} \in B_{F_{i}}} \frac{r\left(P_{j}\right)}{\left|P_{j}\right|}
$$

\subsubsection{Average weighted degree}

O valor de grau médio, $\langle k\rangle$, para uma rede $G$, é definido como:

$$
<k>(G)=\sum_{\forall v \in V} \frac{k(v)}{|V|}
$$

Para o caso ponderado deve-se considerar para cada vértice o valor da carga.

\subsection{Datasets e método}

Datasets consistem em estatísticas disponibilizadas pelo U.S. Department of Transportation (US DOT), entre os anos de 2004 e 2014, para o sistema doméstico de transporte aéreo dos Estados Unidos, contendo dados para cada um dos voos, sendo: (i) aeroporto de origem e de destino; (ii) milhas voadas, (iii) número de passageiros, (iv) volume de carga transportada (mercadorias e carga postal), (v) mês e ano dos voos, dentre outros.

Vale ressaltar que, como estudo de caso, foram selecionados os voos domésticos dos Estados Unidos, dado que a disponibilidade e a facilidade de obtenção de dados são mais amplas, permitindo, então, uma aplicação mais assertiva quanto ao métodolo proposto. Como o dataset possui voos para todos os estados americanos a análise foi realizada para todos eles, diferentemente do recorte tradicional em estudos sobre transporte aéreo onde apenas os 48 estados "continentais" ou "contíguos" são considerados.

Fez-se uma estratificação do conjunto de dados para refletir o segmento do transporte aéreo de cargas (somente bens e mercadorias, excluindo a carga postal), especificamente os voos realizados por Cargo Aircraft. Foram criadas 4 redes para a análise, como mostra a seguir:

- GT20 ou Top20: Constituída pelas 20 empresas operadoras de carga aérea que mais transportaram carga dentro de cada ano considerado, representando pelo menos $90 \%$ do volume total de carga transportada em cada ano, de 2004 a 2014;

- GFedex ou Fedex: Constituída exclusivamente por voos operados pela empresa Federal Express (Fe$\mathrm{dEx}$ ), considerada a maior operadora nesse segmento nos Estados Unidos em termos do volume total de carga transportada entre os anos de 2004 e 2014. Em 2014 operou em torno de $53 \%$ do volume total de carga transportada no ano;
- GUPS ou UPS: Constituída exclusivamente por voos operados pela empresa United Parcel Service (UPS), considerada a segunda maior operadora para os EUA em termos do volume total de carga transportada entre os anos de 2004 e 2014. Juntamente com a Fedex, transportaram acima de $77 \%$ do volume total de carga relativo ao ano de 2014;

- GT18 ou Top18: Constituída pela rede GT20, excluindo-se a Fedex e a UPS.

Como ferramenta de apoio ao cálculo das métricas e plotagem dos grafos utilizou-se o RStudio e o Gephi.

\subsection{Análise e resultados}

Com a estruturação das redes, a aplicação das métricas relacionadas aos métodos de Análise de Redes Sociais e Teoria de Grafos, bem como com base nas contribuições obtidas na literatura existente, foi possível identificar as principais características do modo aeroviário relacionado ao transporte exclusivo de cargas nos Estados Unidos e, preliminarmente, avaliar a evolução dessas redes ao longo do período considerado. Importante observar que a análise foi realizada primordialmente sobre a rede $G_{T 20}$, a qual representa neste estudo a integralidade dessa modalidade de transporte. Nos casos oportunos, foi realizada a comparação com as demais redes $G_{T 18}, G_{F e d e x}$ e $G_{U P S}$. Portanto, quando o artigo se referir de forma genérica ao sistema de transporte aeroviário de carga, estará se referindo à rede $G_{T 20}$, relativa ao ano de 2014.

Antes da apresentação das métricas de rede, o comportamento qualitativo da quantidade de carga transportada pode servir como base para algumas análises. De 2004 a 2014 a quantidade transportada, de acordo com Boeing (2014) e pelos dados do dataset analisado, diminuiu principalmente no período da crise financeira, não atingindo em 2014 os valores de 2004. Além disso, conforme apontado por Donatelli (2012) e pelos dados do dataset analisado, a maior parte do transporte de carga (aprox. 90\%) é realizada por meio de voos exclusivos de carga.

Cabe notar também que a quantidade transportada domesticamente nos EUA entre 2007 e 2013 aumentou (BTS 2, 2016), fato influenciado pelo aumento da quantidade transportada pelo modo rodoviário, o qual representa aproximadamente $75 \%$ do volume transportado nesse país. A Tabela 1 mostra a quantidade transportada em milhões de toneladas nos anos de 2007 e 2013 pelos diferentes modos de transporte (BTS 2, 2016).

A estrutura relacionada à operação do transporte de cargas nos EUA é composta por uma rede formada por 199 aeroportos. Trata-se de um subconjunto do sistema completo, o qual inclui passageiros e carga e é formado por cerca de 850 aeroportos.

Tabela 1. Quantidade transportada em milhões de toneladas nos Estados Unidos 2007 2013

\begin{tabular}{l|c|c|c|c|c|c|c}
\hline Total & Domestic & Exports & Imports & Total & Domestic & Exports & Imports \\
\hline Truck & 12778 & 12587 & 95 & 97 & 13955 & 13732 & 120 \\
\hline Rail & 19 & 1745 & 61 & 93 & 1858 & 1681 & 82 \\
\hline Water & 950 & 504 & 65 & 381 & 808 & 410 & 89 \\
\hline Air, air \& truck & 13 & 3 & 4 & 6 & 15 & 3 & 5 \\
\hline $\begin{array}{l}\text { Multiple modes } \\
\text { \& mail }\end{array}$ & 1429 & 433 & 389 & 606 & 1554 & 459 & 559 \\
\hline Pipeline & 1493 & 1314 & 4 & 175 & 1539 & 1391 & 11 \\
\hline Other unknown & 316 & 266 & 36 & 14 & 333 & 274 & 47 \\
\hline Total & $\mathbf{1 8 , 8 7 9}$ & $\mathbf{1 6 , 8 5 1}$ & $\mathbf{6 5 5}$ & $\mathbf{1 , 3 7 2}$ & $\mathbf{2 0 , 0 6 3}$ & $\mathbf{1 7 , 9 5}$ & $\mathbf{9 1 4}$ \\
\hline
\end{tabular}


Por outro lado, quando comparada com outras redes de transporte de carga e passageiros no mundo, como a do Brasil que é formada por 120 aeroportos (Couto et al., 2015), a rede $G_{T 20}$ mostra-se consideravelmente superior. A Figura 1 apresenta a quantidade de aeroportos para cada rede específica.

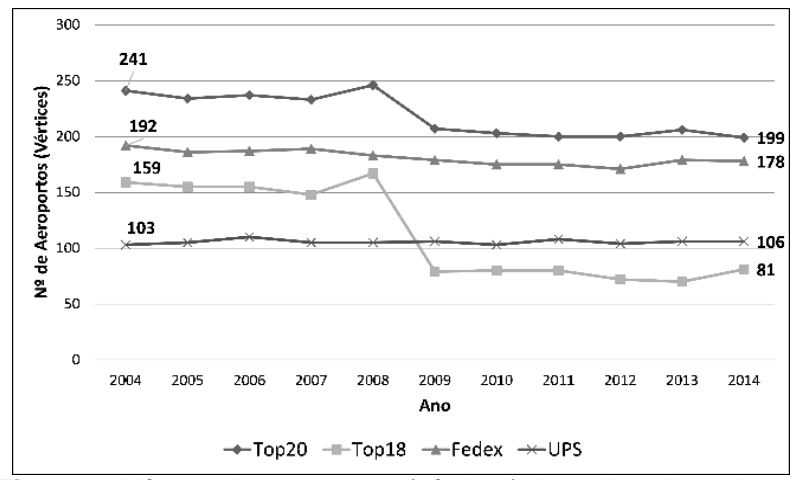

Figura 1. Número de aeroportos (vértices) de cada rede ao longo de 10 anos

É possível observar, na Figura 1, uma gradativa diminuição dos vértices ao longo do período, principalmente para a rede $G_{T 18}$, o que poderia indicar uma possível concentração das operações pelas empresas aéreas em uma quantidade menor de aeroportos. Os dados de número de aeroportos cobertos pela rede $G_{T 20}$ suporta a hipótese de que a crise financeira de 2008 afetou de maneira geral a quantidade de nós da rede, conforme apontam Boeing (2014) e Donatelli (2012) sobre a relação entre crescimento econômico e transporte aéreo de cargas. A rede $G_{T 18}$ é afetada de maneira mais intensa do que as redes das duas maiores companhias, $G_{F e d e x}$ e $G_{U P S}$, sendo que estas podem oferecer preços mais competitivos devido à economias de escala além de terem condições financeiras mais favoráveis, assim como operações integradas, para enfrentamento de períodos de recessão (Onghena, 2013; Morning Star, 2016).

Esse fato pode auxiliar a elaboração de regulações para evitar a concentração excessiva do mercado durante períodos de aguda crise econômica, a exemplo da vivenciada no Brasil entre os anos de 2014 a 2016. Analisando-se os dados do transporte aéreo como um todo, incluindo o transporte combinado e de passageiros, o número de aeroportos também diminuiu no período estudado (BTS 1, 2016), o que colabora para a hipótese de que a crise financeira afetou o número de aeroportos cobertos de maneira geral e não apenas de um segmento específico.

Pode-se notar também que a rede completa possui 21 aeroportos a mais que a rede da Fedex no ano de 2014, o que indica que essa empresa possui cobertura quase que total dos vértices do grafo que representa o transporte de carga.

A Figura 2 indica uma tendência geral de queda, com exceção da Fedex que retornou ao valor de 2004 e à UPS que praticamente não variou, do valor do grau médio de cada aeroporto. Tal fato pode suportar a hipótese de que além do menor número de aeroportos cobertos, o número de destinos e origens de cada nó também foi reduzido devido à crise econômica, principalmente para a rede $\mathrm{G}_{T 18}$. Ainda conforme a Figura 2, cada aeroporto do modo aéreo de cargas, $G_{T 20}$, conecta-se em média com outros 15 aeroportos, representando $7,5 \%$ do total de aeroportos existentes na rede.

Outra característica das redes analisadas é a de que o número de ligações de entrada é similar (aproximadamente entre 1 e 1.5 grau) ao de ligações de saída, o que pode evidenciar a dinâmica de ida e volta entre os centros de distribuição principais e os aeroportos próximos aos consumidores mencionados por Donatelli (2012). Ao se analisar a distribuição do grau de entrada e saída dos vértices nota-se um número maior de aeroportos com poucas conexões (grau de até 10) e um número reduzido de aeroportos com muitas conexões (grau maior que 200). Esse padrão se aproxima mais de topologias centralizadas, como redes tipo estrela, do que as circulares, as quais apresentam distribuição mais uniforme do valor de grau.

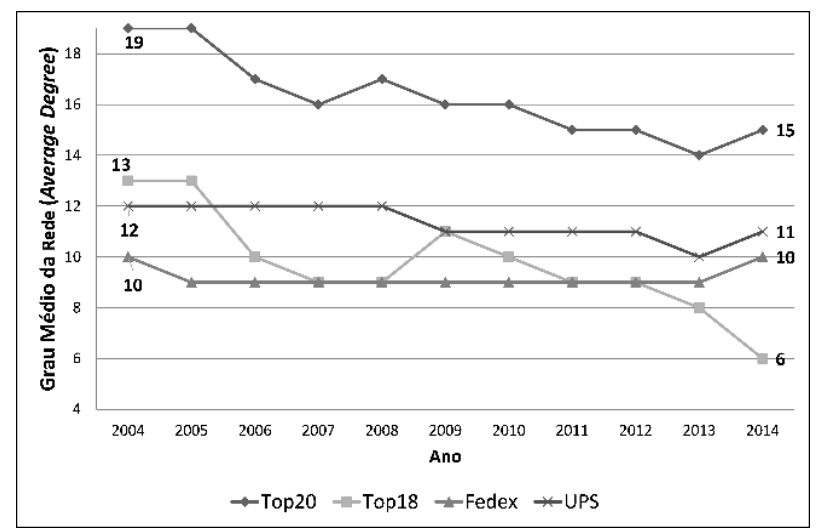

Figura 2. Grau médio (average degree) de cada rede

O transporte aéreo de carga necessita em média passar por outros três aeroportos para chegar no destino final, sendo que o caminho mais curto médio (average shortest path) calculado para a modalidade aérea de cargas é de 2,54. A estrutura operada pela empresa UPS é a que apresenta menor número de "saltos" exigidos para que a carga chegue a sua destinação (o caminho mais curto médio é de 2,08). A Figura 3 apresenta os resultados de average shortest path.

Ainda em consequência da diminuição do número de nós e do grau médio da rede $G_{T 18}$, o caminho médio aumentou chegando a 3,25. Um valor médio maior para se chegar ao destino pode sugerir também custos maiores de transporte, o que pode trazer ainda mais pressão financeira para as empresas da rede $\mathrm{G}_{T 18}$, as quais já se encontram mais afetadas pelo cenário de diminuição de demanda.

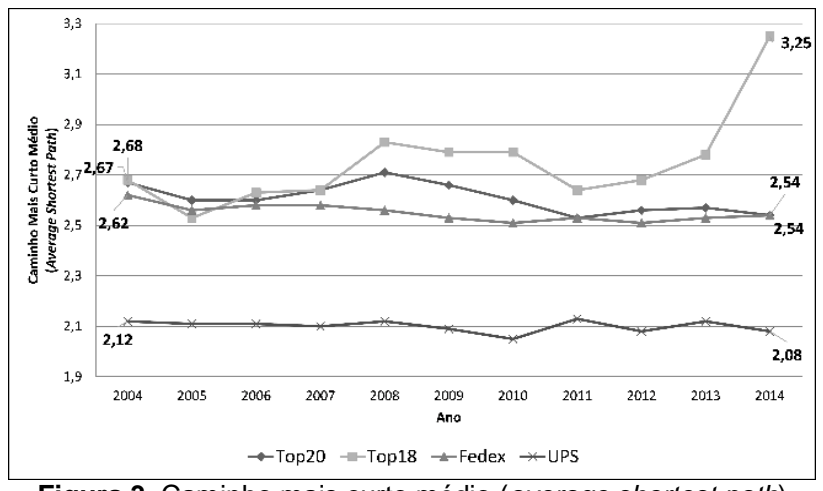

Figura 3. Caminho mais curto médio (average shortest path) 


\begin{tabular}{lccccccccccc}
\multicolumn{10}{c}{ Tabela 2. Diâmetro } \\
\hline Top20 & $\mathbf{2 0 0 4}$ & $\mathbf{2 0 0 5}$ & $\mathbf{2 0 0 6}$ & $\mathbf{2 0 0 7}$ & $\mathbf{2 0 0 8}$ & $\mathbf{2 0 0 9}$ & $\mathbf{2 0 1 0}$ & $\mathbf{2 0 1 1}$ & $\mathbf{2 0 1 2}$ & $\mathbf{2 0 1 3}$ & $\mathbf{2 0 1 4}$ \\
Top18 & 7 & 6 & 5 & 7 & 8 & 9 & 7 & 7 & 6 & 7 & 6 \\
Fedex & 7 & 6 & 7 & 7 & 8 & 8 & 7 & 6 & 6 & 6 & 9 \\
UPS & 5 & 5 & 6 & 6 & 6 & 6 & 6 & 6 & 6 & 6 & 6 \\
\hline
\end{tabular}

Ainda, conforme os resultados para o Diâmetro exibidos na Tabela 2, a viagem mais longa do modo aeroviário de cargas envolve a passagem da carga por 6 aeroportos. No caso da rede para as empresas menores, $G_{T 18}$, a viagem mais longa passa por 9 aeroportos em 2014. A variação de diâmetro de 6 para 9 nessa rede em apenas um ano pode ser reflexo da redução dos nós (aeroportos) e das conexões desses nós, fruto do encolhimento dessas redes em função da queda de demanda. A redução do número de aeroportos servidos, notadamente os menores, devido à crise de 2008 pode ser observado em DOT (2012).

Os resultados obtidos na análise referente ao período de 2004 a 2014, os quais estão refletidos nos gráficos deste artigo, apresentam maior variação para rede $G_{T 18}$ no ano de 2008.

O Coeficiente de Agrupamento médio (clustering coefficient) para a modalidade aérea é de 0,59, significando que em média 59\% dos aeroportos vizinhos estão conectados entre si. Esse parâmetro pode variar de zero para redes tipo estrela a um para redes onde os nós vizinhos possuem todas as conexões entre si. No caso da rede $G_{U P S}$, em média $66 \%$ dos nós vizinhos estão conectados entre si. A Figura 4 apresenta os resultados relativos ao Coeficiente de Agrupamento para todas as redes. A maior variação é observada na rede das 18 maiores empresas $\left(G_{T 18}\right)$, a qual aumentou seu coeficiente de agrupamento, após uma queda acentuada no ano de 2008, o que indica que, após a crise, o número menor de aeroportos restantes, apesar de menos ligações (menor grau médio), possui mais conexões entre si, evidenciando que na rede $G_{T 18}$ os aeroportos menos conectados foram excluídos da mesma. Isso suporta mais uma vez a hipótese de que a queda da demanda advinda da crise financeira excluiu alguns aeroportos mais remotos da rede das 18 maiores empresas.

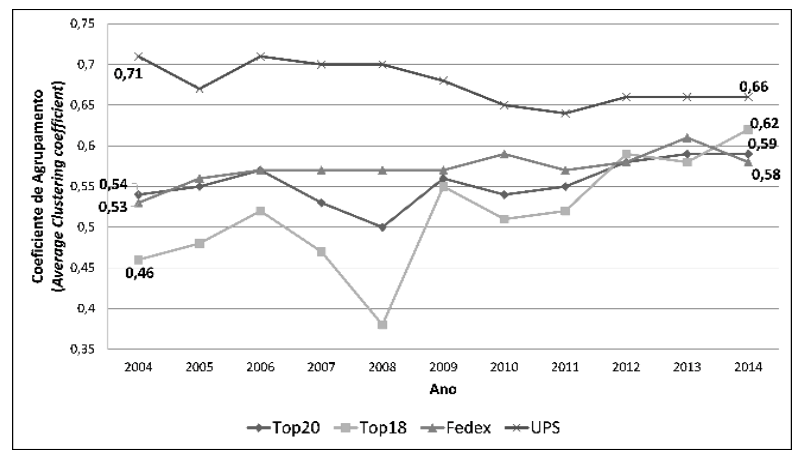

Figura 4. Coeficiente de agrupamento (average clustering coefficient)

A Figura 5 mostra a densidade dos grafos indicando que a rede da empresa UPS é a mais densa, ou seja, possui mais conexões em relação ao total possível de pares origemdestino. A maior variação ocorreu novamente na rede $\mathrm{G}_{T 18}$, onde o aumento da densidade pode evidenciar fato semelhante ao do coeficiente de agrupamento, ou seja, os aeroportos após a crise possuem mais conexões em relação ao total de conexões possíveis da rede. A exceção a essa explicação ocorre no último ano, onde a exclusão de algumas arestas (conexões) afetou o número de conexões.

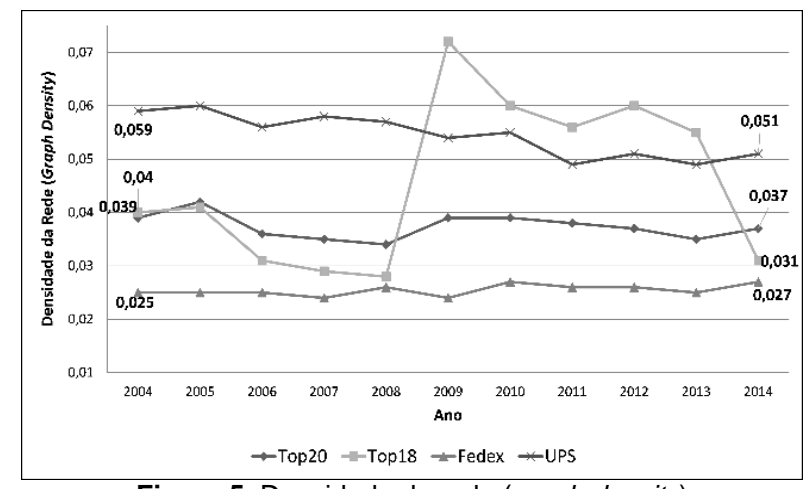

Figura 5. Densidade da rede (graph density)

A Tabela 3 e a Tabela 4 a seguir apresentam os resultados relacionados aos conceitos de centralidade, betweenness e closeness. Apenas os valores de 2014 são apresentados para evidenciar a importância relativa de cada rede, complementando as métricas mostradas acima. Por intermédio desses conceitos é possível observar em cada rede específica quais os aeroportos que desempenham a maior relevância nas operações das empresas aéreas. A Tabela 3 permite observar os 10 aeroportos mais relevantes e que exercem a função de intermediação para os demais aeroportos da rede. Nota-se que a rede com as 20 maiores empresas possui como nós com maior intermediação os dois principais aeroportos das duas maiores empresas, o que evidencia a centralidade desses vértices quando se analisa a rede de transporte de carga nos Estados Unidos. O maior grau de centralidade dos aeroportos das duas maiores empresas não implica necessariamente na utilização dessas instalações na operação das 18 outras aéreas.

A Tabela 4 mostra os aeroportos mais importantes em termos de proximidade, ou seja, quais aeroportos originam as viagens que percorrem a menor distância para atingir os demais nós da rede. Pode-se ver que Memphis e Louisville, bases centrais para as empresas Fedex e UPS, representam os nós com maior centralidade de proximidade das respectivas redes, incluindo a rede com as 20 maiores empresas, suportando a hipótese de que as duas companhias desempenham papel relevante no setor nos EUA.

Os valores dos graus médios ponderados por carga para as redes T20, T18, Fedex e UPS em milhões de libras durante 2014 são, respectivamente, 216, 98, 126 e 116. Nota-se que a quantidade média transportada por aeroporto é maior para a rede Top20, pois essa compreende todas as maiores empresas. Já as redes Fedex e UPS apresentaram valores similares aos das 18 demais aéreas, o que pode evidenciar o fato de que as duas maiores empresas do setor nos Estados Unidos transportam por aeroporto quantidades similares às outras 18 , porém como as primeiras possuem um grau médio maior, transportam de maneira absoluta mais carga do que as últimas. 
MALERE, J.P.; MINAS, V.; BORILLE, G.M.R.

Tabela 3. Os 10 aeroportos mais importantes em termos de betweenness (2014)

\begin{tabular}{|c|c|c|c|c|}
\hline & $\begin{array}{c}\text { T20 } \\
\text { (Betweenness - Aeroporto) } \\
\end{array}$ & $\begin{array}{c}\text { T18 } \\
\text { (Betweenness - Aeroporto) }\end{array}$ & $\begin{array}{c}\text { Fedex } \\
\text { (Betweenness - Aeroporto) } \\
\end{array}$ & $\begin{array}{c}\text { UPS } \\
\text { (Betweenness - Aeroporto) }\end{array}$ \\
\hline $\mathbf{1}^{\circ}$ & 0,349-Memphis, TN & 0,2614-Cincinnati, $\mathrm{OH}$ & 0,6076-Memphis, TN & 0,8245-Louisville, KY \\
\hline $2^{\circ}$ & 0,1889-Louisville, KY & 0,1741-Anchorage, AK & 0,1025 -Indianapolis, IN & 0,0317-Philadelphia, PA \\
\hline $3^{\circ}$ & 0,054-Indianapolis, IN & 0,0734-Los Angeles, CA & 0,0433-Anchorage, AK & 0,0294-Ontario, CA \\
\hline $4^{\circ}$ & 0,048-Anchorage, $\mathrm{AK}$ & 0,0716-Wichita, KS & 0,0419-Ontario, CA & 0,0234-Honolulu, HI \\
\hline $5^{\circ}$ & 0,0462-Ontario, CA & 0,0706 -Everett, WA & 0,0303-Oakland, CA & 0,0229-Rockford, IL \\
\hline $6^{\circ}$ & 0,0295-Honolulu, HI & 0,0702-New York, NY & 0,0252 -Great Falls, MT & 0,0189-Anchorage, AK \\
\hline $7^{\circ}$ & 0,0219-Portland, OR & 0,0691-Louisville, KY & 0,0236-Honolulu, HI & 0,0109-Reno, NV \\
\hline $8^{\circ}$ & 0,0214-Great Falls, MT & $\begin{array}{l}, 0592-D a l l a s / \text { Fort } \\
\text { Worth, TX }\end{array}$ & $\begin{array}{l}0,0229-\text { Dallas/Fort } \\
\text { Worth, TX }\end{array}$ & $\begin{array}{l}0,0103 \text {-Dallas/Fort } \\
\text { Worth, TX }\end{array}$ \\
\hline $9^{\circ}$ & 0,0205-Oakland, CA & 0,0554-Miami, FL & $\begin{array}{c}\text { 0,0205-Greensboro/High Point, } \\
\text { NC }\end{array}$ & 0,0095-Richmond, VA \\
\hline $10^{\circ}$ & 0,0198-Cincinnati, $\mathrm{OH}$ & 0,0548-Honolulu, HI & 0,0205-Spokane, WA & 0,0094-Manchester, $\mathrm{NH}$ \\
\hline
\end{tabular}

Tabela 4. Os 10 aeroportos mais importantes em termos de closeness (2014)

\begin{tabular}{|c|c|c|c|c|}
\hline & $\begin{array}{c}\text { T20 } \\
\text { (Closeness - Aeroporto) }\end{array}$ & $\begin{array}{c}\text { T18 } \\
\text { (Closeness - Aeroporto) }\end{array}$ & $\begin{array}{c}\text { Fedex } \\
\text { (Closeness - Aeroporto) }\end{array}$ & $\begin{array}{c}\text { UPS } \\
\text { (Closeness - Aeroporto) }\end{array}$ \\
\hline $\mathbf{1}^{\circ}$ & 0,1354-Memphis, TN & 0,0485-Cincinnati, $\mathrm{OH}$ & 0,7468 -Memphis, TN & 0,9633-Louisville, KY \\
\hline $2^{\circ}$ & 0,1329 -Louisville, KY & 0,0482 -Anchorage, $\mathrm{AK}$ & 0,5728 -Indianapolis, IN & 0,5932-Philadelphia, PA \\
\hline $\mathbf{3}^{\circ}$ & 0,129-Indianapolis, IN & 0,0481-New York, NY & 0,5014-Dallas/Fort Worth, TX & 0,5833-Rockford, IL \\
\hline $4^{\circ}$ & 0,1262-Los Angeles, CA & 0,0479-Miami, FL & 0,4972-Los Angeles, CA & 0,5801-Ontario, $\mathrm{CA}$ \\
\hline $5^{\circ}$ & 0,1258-Oakland, CA & 0,0479-Chicago, IL & 0,4972-Oakland, CA & 0,5707-Dallas/Fort Worth, TX \\
\hline $6^{\circ}$ & 0,1258-Philadelphia, PA & 0,0479-Los Angeles, CA & 0,481-Newark, NJ & 0,5385-Des Moines, IA \\
\hline $7^{\circ}$ & 0,1257-Dallas/Fort Worth, TX & 0,0477-Dallas/Fort Worth, TX & 0,4646-Ontario, CA & 0,5303 -Portland, OR \\
\hline $\mathbf{8}^{\circ}$ & 0,1255-Newark, NJ & 0,0477-Louisville, KY & 0,4621-Phoenix, AZ & 0,5303-Phoenix, AZ \\
\hline $9^{\circ}$ & 0,1255-Dallas/Fort Worth, TX & 0,0476-Philadelphia, PA & 0,4585-Portland, OR & 0,5276-Denver, CO \\
\hline $10^{\circ}$ & 0,1255 -Anchorage, $\mathrm{AK}$ & 0,0475 -Houston, TX & 0,4585 -Seattle, WA & 0,525 -Anchorage, AK \\
\hline
\end{tabular}

Tabela 5. Os 10 aeroportos mais importantes em termos de PageRank (2014)

T20

(Page Rank-Aeroporto) $\quad$ (Page Rank - Aeroporto)

\begin{tabular}{|c|c|c|c|c|}
\hline & (Page Rank-Aeroporto) & (Page Rank - Aeroporto) & (Page Rank - Aeroporto) & (Page Rank - Aeroporto) \\
\hline $\mathbf{1}^{\circ}$ & 0,069-Memphis, TN & 0,0827- Cincinnati, $\mathrm{OH}$ & $0,131-$ Memphis, TN & 0,182- Louisville, $\mathrm{KY}$ \\
\hline $2^{\circ}$ & 0,0574-Louisville, $\mathrm{KY}$ & 0,0365 - Anchorage, $\mathrm{AK}$ & 0,064- Indianapolis, IN & 0,045- Philadelphia, PA \\
\hline $3^{\circ}$ & 0,0337- Indianapolis, IN & 0,0355- Miami, FL & $\begin{array}{l}\text { 0,026- Dallas/Fort } \\
\text { Worth, TX }\end{array}$ & 0,042-Rockford, IL \\
\hline $4^{\circ}$ & 0,0192- Ontario, CA & 0,0314- Louisville, $\mathrm{KY}$ & 0,025 - Newark, NJ & 0,037 - Ontario, CA \\
\hline $5^{\circ}$ & 0,0178- Cincinnati, $\mathrm{OH}$ & 0,0269- Los Angeles, CA & 0,022- Oakland, CA & $\begin{array}{l}\text { 0,030- Dallas/Fort } \\
\text { Worth, TX }\end{array}$ \\
\hline $6^{\circ}$ & 0,0173- Philadelphia, PA & 0,0264- Honolulu, HI & 0,015- Los Angeles, CA & 0,024- Des Moines, IA \\
\hline $7^{\circ}$ & 0,0164- Newark, NJ & 0,0254- Chicago, IL & 0,014- Ontario, CA & 0,016- Portland, OR \\
\hline $8^{\circ}$ & 0,0159- Oakland, CA & 0,0251- Charleston, SC & 0,012 - St. Louis, MO & 0,014- Sacramento, CA \\
\hline $9^{\circ}$ & 0,0158- Anchorage, $\mathrm{AK}$ & 0,0239 - Everett, WA & 0,012- Anchorage, $\mathrm{AK}$ & 0,014- Seattle, WA \\
\hline $10^{\circ}$ & $\begin{array}{c}\text { 0,0157-Dallas/Fort } \\
\text { Worth, TX }\end{array}$ & 0,0223- New York, NY & 0,012- Nashville, TN & 0,014- Oakland, CA \\
\hline
\end{tabular}

As Figuras de 6 e 7 mostram mapas com os grafos não-ponderados de duas redes para o ano de 2014. A Figura 6 mostra a rede da Fedex, sendo que o nó com maior grau se encontra em Memphis (sudoeste do estado americano do Tennessee).

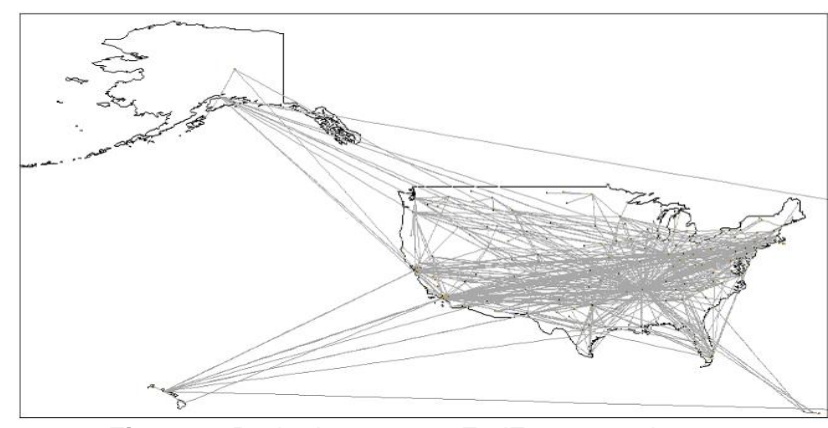

Figura 6. Rede da empresa FedEx no ano de 2014

A Figura 7 mostra a rede com as 18 maiores empresas, onde se pode visualizar o número menor de aeroportos e o menor grau médio em comparação com a rede da Fedex.

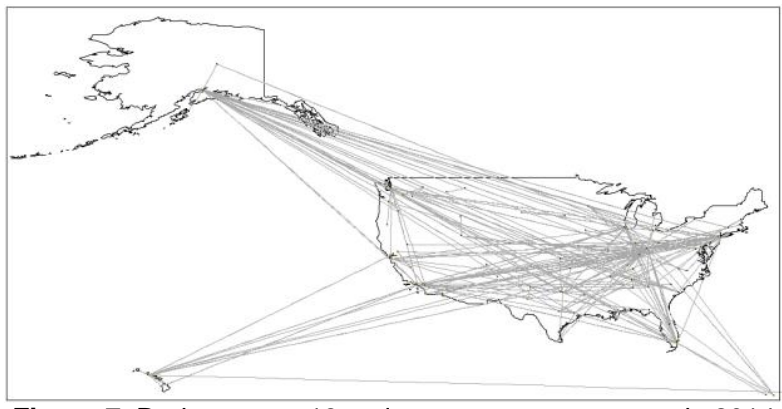

Figura 7. Rede com as 18 maiores empresas no ano de 2014

\section{CONSIDERAÇÕES FINAIS}

O presente trabalho apresentou as características da rede de transporte aéreo de carga dos Estados Unidos entre os anos de 2004 e 2014 através da Análise de Redes Sociais e Teoria de Grafos. Quatro diferentes redes compreendendo as 20 maiores empresas em termos de carga foram comparadas. De maneira geral, a hipótese de que tais métricas podem auxiliar em estudos econômicos na área de logística e de que há preponderância do sistema de transporte de carga 
exercida por um reduzido número de grandes empresas, foi suportada pela relação entre as métricas de rede e o comportamento econômico do período estudado, principalmente quanto à redução do produto interno bruto após a crise financeira de 2008. Há evidências de que as duas maiores empresas do setor, Fedex seguida pela UPS, influenciam a topologia da rede de carga aérea pela expressiva cobertura de aeroportos e rotas. Além disso, essa rede apresenta valores maiores de coeficiente de clustering e de número de aeroportos que outras grandes redes, como a de transporte de passageiros do Brasil. Refletindo o grande número de voos dos aeroportos principais das duas grandes empresas de carga dos EUA, Memphis e Louisville apresentam os maiores valores de proximidade (closeness) e intermediação (betweenness). A rede com as 18 maiores empresas apresenta maior variação e maior sensibilidade no que se refere às métricas de rede em um cenário com grande variação de demanda como o ocorrido em 2008. Como estudos futuros poder-se-ia analisar mais hipóteses com relação ao mesmo dataset utilizado, além de outros tipos de transporte, como o Combi. Além disso, as redes de outros países, como as o Brasil, poderiam ser investigadas e comparadas.

\section{REFERÊNCIAS}

Batti, D. A. B., \& Faria, C. A. (2011). Transporte aéreo-uma alternativa ao transporte rodoviário de cargas. Journal of Transport Literature, 5(2), 92-105.

BOEING (2014). World Air Cargo forecast - 2014-15. <http://www.boeing.com/resources/boeingdotcom/commercial/a bout-our-market/cargo-market-detail-wacf/downloadreport/assets/pdfs/wacf.pdf $>$. Acessado em 20 de novembro de 2015.

Bowen, J. T. (2004). The geography of freighter aircraft operations in the Pacific Basin. Journal of Transport Geography, 12(1), 1-11. DOI: 10.1016/S0966-6923(03)00024-3.

Brownrigg R (2012) Package 'maps'. Disponível em <http://cran.r-project.org/web/packages/maps/>. Acessado en 20 de março de 2016.

BTS 1 (2016). Number of U.S. Airports.

<http://www.rita.dot.gov/bts/sites/rita.dot.gov.bts/files/publicatio ns/national_transportation_statistics/html/table_01_03.html>.

Acessado em 01 de julho de 2016.

BTS 2 (2016). Weight of shipments by transportation mode .<http://www.rita.dot.gov/bts/sites/rita.dot.gov.bts/files/dat a_and_statistics/by_subject/freight/freight_facts_2015/chapter2/t able2_1>.Acessado em 01 de julho de 2016.

Cerqueira, C. H. Z., de Souza Costa, J. M., \& de Araujo Carvalho, D. M. (2014). Aplicação de Análise de Redes Sociais em uma Cadeia de Suprimentos de uma Empresa do Setor Elétrico Brasileiro. Sistemas \& Gestão, 9(4), 418-429. DOI: 10.7177/sg.2014.v9.n4.a1

Cook, G. N., \& Goodwin, J. (2008). Airline Networks: A Comparison of Hub-and-Spoke and Point-to-Point SystemsAirline Networks: A Comparison of Hub-and-Spoke and Point-to-Point Systems. Journal of Aviation/Aerospace Education \& Research, 17(2), 1.
Couto, G. S., Silva, A. P. C. D., Ruiz, L. B., \& Benevenuto, F. (2015). Structural properties of the Brazilian air transportation network. Anais da Academia Brasileira de Ciências, 87(3), 16531674. DOI: 10.1590/0001-3765201520140155.

Csardi G, Nepusz T (2006): The igraph software package for complex network research, InterJournal, Complex Systems 1695.

De Nooy, W., Mrvar, A., \& Batagelj, V. (2011). Exploratory social network analysis with Pajek (Vol. 27). Cambridge University Press. DOI: 10.1017/CBO9780511806452.

Donatelli, D. J. (2012). Evolution of US air cargo productivity (Master of Science Dissertation, Massachusetts Institute of Technology).

DOT (2012). Aviation industry Performance - A review of the aviation industry, 2008-2011.

<https://www.oig.dot.gov/sites/default/files/Aviation\%20Industr y\%20Performance\%5E9-24-12.pdf $>$. Acessado em 28 de Agosto de 2016.

Freeman, L. C. (1979). Centrality in social networks conceptual clarification. Social networks, 1(3), 215-239. DOI:

10.1016/0378-8733(78)90021-7.

Grandjot, H. H., Roessler, I., \& Roland, A. (2007). Air Cargo Guide: An introduction to the air cargo industry. Huss-Verlag.

Helmig, C. (2005). Welcome the Change. Flying Fresh-The perishable air cargo magazine, 1,5 .

Morning Star (2016). FedEx is one of two titans in private U.S. parcel shipping. We don't expect this to change. <http://analysisreport.morningstar.com/stock/research/creport?t=XNYS:FDX\&region $=$ usa\&culture $=$ en US\&productcode=MLE\&cur=> . Acessado em 28 de Agosto de 2016.

Newman, M. E. (2003). The structure and function of complex networks. SIAM review, 45(2), 167-256. DOI: $10.1137 / \mathrm{S} 003614450342480$.

Newman, M. E. (2004). Analysis of weighted networks. Physical Review E,70(5), 056131. DOI:10.1103/PhysRevE.70.056131

Onghena, E. (2013). From cost structure to strategy: the impact of the cost structure on the strategic behavior of integrators $(\mathrm{PhD}$ thesis Faculty of Applied Economic Sciences, University of Antwerp).

Page, L., Brin, S., Motwani, R., \& Winograd, T. (1998). The PageRank citation ranking: bringing order to the Web. Tech. Rep., Stanford Digital Library Technologies Project. DOI: 10.1.1.31.1768

Reka A., Barabási A.L. (2002). Statistical mechanics of complex networks. Rev Mod Phys 74:47-97.

Scholz, A. B. (2012). Network structures of cargo airlines-an empirical and a modelling approach. KIT Scientific Publishing.

Scott, J. (1991). Networks of corporate power: A comparative assessment. Annual Review of Sociology, 181-203.

Scott, J., \& Carrington, P. J. (2014). The SAGE handbook of social network analysis. SAGE publications. DOI: $10.4135 / 9781446294413$.

Wasserman, S., \& Faust, K. (1994). Social network analysis: Methods and applications (Vol. 8). Cambridge university press. 